\title{
Mixing it up
}

Nature Astronomy is uniquely positioned to publish a variety of excellent scientific research alongside commentary, opinion and editorial content on the topics that matter most to the astronomy and planetary science communities. We are committed to promoting diversity in peer review and the voices we feature.

W elcome to the 33 rd issue of Nature Astronomy: almost one third of the way to a century. When Nature Astronomy launched in January 2017, we were something of an unknown quantity to most of our community. Astronomers and astrophysicists did not have a specialist Nature Research journal to submit their work to, although some astronomy research was published in Nature Physics. Planetary scientists might have been familiar with Nature Geoscience, but fields like the study of exoplanets, a multidisciplinary subject born out of the merging of astronomy and planetary science, had no natural home. Nature Astronomy fills that gap, and welcomes all of astronomy, astrophysics and planetary science, and their crossovers. Hopefully, now that Nature Astronomy has been accepting submissions for three years, and our editors have been trying to meet and interact with as many of you as possible, you know us, what we're looking to publish, and how we work. If you feel that is not the case, please get in touch; we'd love to hear from you and tell you more.

We hope that what you know of us includes our commitment to improving diversity and inclusion in our community in any way we can. In our sixth issue we published a Focus on 'Gender equity in astronomy', and ( ${ }^{*}$ spoiler alert $\left.{ }^{*}\right)$ with another Focus coming up, we will be revisiting and broadening that theme. In the last few years, we have been working with the European Astronomical Society to help support and organize the 'Inclusion day' at their European Week of Astronomy and Space Science meetings. We were very pleased to sponsor a similar initiative at the Royal Astronomical Society's National Astronomy Meeting, and we are looking forward to the International Astronomical Union's (IAU's) first symposium on 'Equity, Diversity and Inclusion' in November, which we are also sponsoring.

In our peer-review and publication process, we want to also be as inclusive and equitable as possible. In our choice of reviewers, we try to be mindful of the gender, seniority and geographical location of our invitees. Our first-choice referees understandably are not always available, and we encourage our declining reviewers to

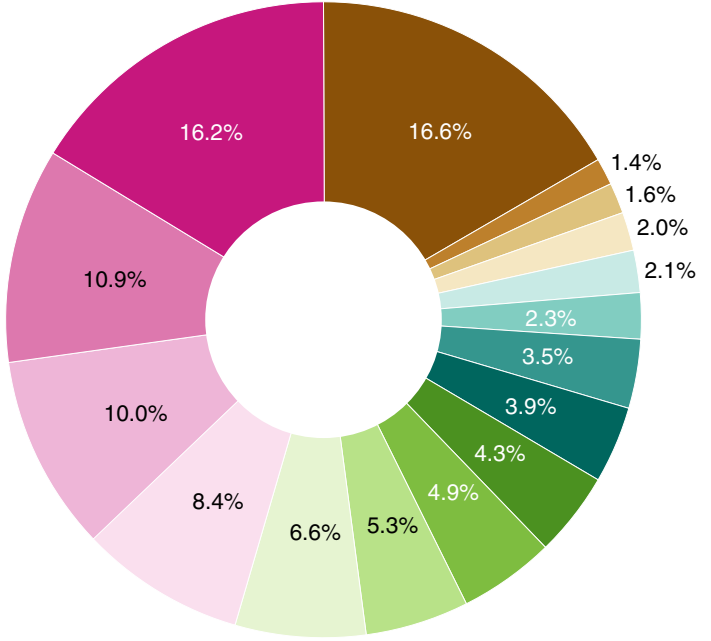

Planets and moons

Stars

Galaxies and clusters

High-energy astrophysics

Interstellar medium

Cosmology

Exoplanets

Small bodies

Early Solar System

Computational astrophysics

Particle astrophysics

Astrophysical disks

Meteoritics

Solar physics

Astronomical instrumentation

Other

"help us increase the diversity of our referees if at all possible" (an extract from our review request email) when suggesting alternatives. Looking at all of the manuscripts sent out for peer review up until the end of June 2019, at least $21 \%$ of our reviewers have been women (that is, have a first name that has a strong association with people presenting as female). Given that we could not confidently assign a gender to some first names (for example, unisex or rare names), this figure in reality is likely to be closer to $25 \%$. This number is far from 50:50 parity, but it is double the percentage of female corresponding authors who submit manuscripts to Nature Astronomy ( 11\%), and it exceeds the percentage of women faculty in astronomy (as reported by the American Institute of Physics, for example, in the country where most astronomers reside: 19\%; 2014 data) and the percentage of women members of the IAU (18\%; August 2019 data). We understand that women in academia carry out more service tasks than men, and we are especially grateful to the women who choose to accept our requests to review submissions.

We do not collate data on the seniority or geographical location of reviewers, but anecdotally, we try to assign reviewers across a wide range of career stages, from early career to emeritus, and we aim to improve geographical diversity by searching for qualified reviewers from countries outside the main astronomy strongholds in Europe and North America.
Table 1 | Submissions by country

\begin{tabular}{ll} 
Country & \% of total submissions \\
\hline USA & 21.2 \\
China & 16.8 \\
UK & 7.8 \\
Japan & 5.6 \\
India & 5.0 \\
\hline Italy & 3.7 \\
\hline France & 3.5 \\
Germany & 3.4 \\
Canada & 3.1 \\
Rep. Korea & 2.4
\end{tabular}

It has been shown that women submitting manuscripts find it more challenging to have a paper accepted for publication than a man submitting a similar paper. Thankfully this is not the case at Nature Astronomy, and we will work to ensure that the situation stays that way. At Nature Astronomy, the editor's decision to send a manuscript to reviewers or not is independent of the gender of the corresponding author ( $p$-value of 0.234 ; $N=1,133$ manuscripts). Post-review, women corresponding authors have a $6 \%$ higher chance of having their manuscript accepted than male corresponding authors according to the numbers.

In our non-peer-reviewed content we aim to promote diversity in the author list 
and the subject material, particularly when it comes to geography and gender. In addition to our Focuses on gender equity, last year we initiated a living collection on 'Astronomy for development', which features astronomy initiatives and facilities from around the globe, especially in Africa. In terms of gender, women first authors contribute $~ 37 \%$ of our Comments, News \& Views, Mission Controls, Perspectives, Reviews and more.

Geographically, our submitting authors come from all over the world; Table 1 shows the top ten countries, but there is a long tail that encompasses nearly 100 countries - as different as Bulgaria and Botswana, Portugal and Pakistan, Egypt and Ecuador.
In terms of subject material, too, our research papers cover diverse topics, on size scales from large to small, energy scales from high to low, from theory to laboratory to numerical simulation to observation. The pie chart shows the large variety of topics covered by our published research papers in issues to date.

Nature Astronomy, as a journal, is uniquely positioned to offer both contributed and commissioned content for our specialist astronomy and planetary science communities. Our aim is to be a venue for the presentation and discussion of excellent science, and at the same time, a forum for the airing of community issues and celebration of community initiatives. We very much believe that diverse communities make for stronger communities, and that in the past astronomy and planetary science have not been as diverse or as supportive of diversity as they should have been. Our aim is to do what we can to change things for the better, and you can help by pitching ideas for articles or Focuses, talking to and educating our editors at conferences and institute visits, or by becoming a part of our online Astronomy Community.

Published online: 9 September 2019 https://doi.org/10.1038/s41550-019-0898-5 